\title{
Thermodynamics of the vortex liquid in heavy-ion-irradiated superconductors
}

\author{
Cornelis J. van der Beek and Marcin Konczykowski \\ Laboratoire des Solides Irradiés, CNRS-UMR 7642 \& CEA/DSM/DRECAM, Ecole Polytechnique, 91128 Palaiseau cedex, France
}

Luc Fruchter

Laboratoire de Physique des Solides, Bâtiment 510, Université Paris-Sud, CNRS, 91405 Orsay, France

René Brusetti, Thierry Klein, and Jacques Marcus

Laboratoire d'Etudes des Propriétés Electroniques des Solides, Centre National de la Recherche Scientifique, B.P. 166, 38042 Grenoble cedex 9, France

Christophe Marcenat

Département de Recherche Fondamentale sur la Matière Condensée, Service de Physique des Solides Magnétiques et Supraconducteurs, Commissariat à l'Energie Atomique, 17 Avenue des Martyrs, 38054, Grenoble cedex 9, France

(Received 1 April 2005; revised manuscript received 23 September 2005; published 7 December 2005)

\begin{abstract}
It is shown that the large effect of heavy ion irradiation on the thermodynamical properties of the anisotropic superconductor $\mathrm{YBa}_{2} \mathrm{Cu}_{3} \mathrm{O}_{7-\delta}$ extends well into the superconducting fluctuation regime. The presence of the induced amorphous columnar defects shifts the specific-heat maximum at the normal-to-superconducting transition. This effect is similar to that recently put into evidence in cubic $\mathrm{K}_{x} \mathrm{Ba}_{1-x} \mathrm{BiO}_{3}(x \simeq 0.35)$. In both compounds, vortex pinning manifests itself as a sharp angular dependence of the equilibrium torque. In $\mathrm{YBa}_{2} \mathrm{Cu}_{3} \mathrm{O}_{7-\delta}$, pinning by the defects appears at the temperature $T_{C_{p}}^{\max }$ of the specific-heat maximum, well above the magnetic irreversibility line $T_{i r r}(H)$. In isotropic $\mathrm{K}_{x} \mathrm{Ba}_{1-x} \mathrm{BiO}_{3}$, the onset of the pinning-related torque anomaly tracks the onset of the specific-heat anomaly and the irreversibility line. In $\mathrm{YBa}_{2} \mathrm{Cu}_{3} \mathrm{O}_{7-\delta}$, fluctuations of the amplitude of the order parameter (and not vortex line wandering) are ultimately responsible for the vanishing of pinning. In $\mathrm{K}_{x} \mathrm{Ba}_{1-x} \mathrm{BiO}_{3}$, vortex pinning disappears only at the superconducting-to-normal transition. The results indicate that in both compounds, the pinning energy at the "Bose-glass" transition is large with respect to the total free energy gain in the superconducting state. By implication, the mechanism of this latter transition should be reconsidered.
\end{abstract}

DOI: 10.1103/PhysRevB.72.214504

PACS number(s): 74.25.- q, 74.40.+k, 74.62.Dh

\section{INTRODUCTION}

When heated above their irreversibility line, disordered type II superconductors undergo a transition from a "truly superconducting" ensemble of localized vortex lines to a "vortex liquid" of diffusing lines. This transition is most commonly described in terms of a thermal "depinning" of vortex lines from material defects. Typically, thermal wandering of the vortex lines from the defects is thought to become increasingly important as the temperature is raised, until, at the transition, the free energy gain obtained from vortex localization on the defects has dropped to $\sim k_{B} T$.

The case where the defects are columnar amorphous tracks introduced by swift heavy-ion irradiation has attracted much attention, not in the least because the problem becomes particularly tractable theoretically. Using the formal analogy between flux lines and two-dimensional (2D) bosons in a static disorder potential, Nelson and Vinokur ${ }^{1}$ calculated the magnitude of thermal positional excursions, concomitant pinning energies, and the resultant phase diagram. This consists of a low temperature disordered "Bose glass" of localized vortices that gives way to the vortex liquid at the temperature $T_{B G}(B)$ [or induction $B_{B G}(T)$ ]. Model descriptions of the Bose glass-to-liquid transition nearly exclusively rely on the total pinning energy near $T_{B G}(B)$ being small. This can be either because line wandering reduces the single-vortex pin- ning energy, ${ }^{1,2}$ or because vortices vastly outnumber the defects. In both cases, the pinning energy gain is only a small perturbation to the total free energy of the system, and the position of the Bose-glass transition line can be obtained as a shift $^{1-6}$ of the (first order) vortex lattice melting line of the pristine material. ${ }^{7-9}$ This approach has had some success in explaining the observed increase of $B_{B G}$ with defect density $n_{d}$ in $\mathrm{YBa}_{2} \mathrm{Cu}_{3} \mathrm{O}_{7-\delta}$, where it was found that $\partial B_{B G} / \partial T \sim 1$ $+A \Phi_{0}^{1 / 2} n_{d}^{1 / 2}$ (with $\Phi_{0}=h / 2 e$ the flux quantum). ${ }^{2,4}$

Simultaneously, experiments abound indicating that the pinning energy near the Bose-glass transition in cuprate superconductors is not small. Reversible magnetization measurements on heavy-ion-irradiated layered $\mathrm{Bi}_{2} \mathrm{Sr}_{2} \mathrm{CaCu}_{2} \mathrm{O}_{8}$ reveal a large contribution of columnar defect pinning to the free energy. ${ }^{10,11}$ An effect of the ion tracks was measured up into the fluctuation critical regime, ${ }^{10}$ and interpreted in terms of local $T_{c}$ variations induced by the defects. ${ }^{10,12}$ Recent measurements on cubic $(\mathrm{K}, \mathrm{Ba}) \mathrm{BiO}_{3}$ have shown that heavyion irradiation even affects the specific heat $C_{p}$ : the temperature at which the $C_{p}$ jump occurs, signaling the transition to the superconducting state, was found to shift upward with increasing defect density $n_{d}$, and depends on the angle between magnetic field and the track direction. ${ }^{13,14}$ When the magnetic field is aligned with the tracks, superconductivity is enhanced, when the magnetic field is turned away from the tracks, one recovers the behavior of the pristine crystal. As 
TABLE I. Superconducting parameters for the studied compounds.

\begin{tabular}{lcc}
\hline \hline & $\mathrm{YBa}_{2} \mathrm{Cu}_{3} \mathrm{O}_{7-\delta}$ & $\mathrm{K}_{0.35} \mathrm{Ba}_{0.65} \mathrm{BiO}_{3}$ \\
\hline$\lambda$ & $\lambda_{a b}(0)=120 \mathrm{~nm}$ & $\lambda(0)=220 \mathrm{~nm}$ \\
$\xi$ & $\xi_{a b}(0)=1.4 \mathrm{~nm}$ & $\xi(0)=3.8 \mathrm{~nm}$ \\
$\varepsilon_{0}(0)$ & $1.7 \times 10^{-11} \mathrm{Jm}^{-1}$ & $5.2 \times 10^{-12} \mathrm{Jm}^{-1}$ \\
$G i$ & $2 \times 10^{-3}$ & $5 \times 10^{-6}$ \\
$\varepsilon=\lambda_{a b} / \lambda_{c}=\xi_{c} / \xi_{a b}$ & 0.14 & 1 \\
\hline \hline
\end{tabular}

for heavy-ion-irradiated $\mathrm{YBa}_{2} \mathrm{Cu}_{3} \mathrm{O}_{7-\delta}$, transport experiments show that in the vortex liquid, the resistivity remains exponentially small with respect to that of the pristine material. ${ }^{15-18}$ More strikingly, the experiments of Refs. 16-18 reveal an angular dependence of the resistivity, related to vortex pinning by the tracks, that persists up to resistance levels that are $90 \%$ of that in the normal state. In other words, columnar defects affect vortices in the vortex liquid, and up into the fluctuation paraconductivity regime. However, there are no thermodynamic measurements assessing the importance of the pinning energy in this material.

In order to establish the magnitude of the contribution of vortex pinning by columnar defects to the free energy, we have performed measurements of the specific heat and the reversible torque on heavy-ion-irradiated single crystalline $\mathrm{YBa}_{2} \mathrm{Cu}_{3} \mathrm{O}_{7-\delta}$ (Secs. II C and II B). The data are compared to previous specific-heat results ${ }^{13,14}$ and new torque measurements on single crystalline $\mathrm{K}_{x} \mathrm{Ba}_{1-x} \mathrm{BiO}_{3}$. The main difference between the two materials lies in their Ginzburg number $G i \equiv(1 / 2)\left[k_{B} T_{c} / 2 \pi \varepsilon \varepsilon_{0}(0) \xi(0)\right]^{2}$. Here $\varepsilon_{0}(T)$ $=\Phi_{0}^{2} / 4 \pi \mu_{0} \lambda_{a b}^{2}(T)$ is the vortex energy scale, $\lambda_{a b}(T)$ is the penetration depth for currents running perpendicularly to the crystalline anisotropy axis, and $\xi(T)$ the coherence length (for $\mathrm{YBa}_{2} \mathrm{Cu}_{3} \mathrm{O}_{7-\delta}, \xi=\xi_{a b}$, the $a b$-plane coherence length). Since $G i$ for optimally doped $\mathrm{YBa}_{2} \mathrm{Cu}_{3} \mathrm{O}_{7-\delta}$ is two orders of magnitude larger than that of $\mathrm{K}_{x} \mathrm{Ba}_{1-x} \mathrm{BiO}_{3}$ (see Table I), it is conceivable that thermal fluctuations wipe out any strong effect of columnar defects in the vortex liquid phase. On the contrary, we find that the reduced temperature $T_{C_{p}}^{\max }(H) / T_{c}$ at which the specific heat is maximum in $\mathrm{YBa}_{2} \mathrm{Cu}_{3} \mathrm{O}_{7-\delta}$ unambiguously shifts upward with increasing columnar defect density $n_{d}$, as it does in $\mathrm{K}_{x} \mathrm{Ba}_{1-x} \mathrm{BiO}_{3}$. This upward shift can only be accounted for by a large (i.e., not perturbatively small) contribution of pinning by the columnar defects to the free energy. This is in contradiction to the assumptions commonly made in estimating the Bose-glass transition line. ${ }^{1-6}$ In Sec. III B, we estimate the mean-field pinning energy contribution required for the specific-heat shift.

We also obtain the experimental pinning energy directly from reversible torque measurements (Sec. III A). It turns out that the field and temperature dependence of the pinning energy, rather surprisingly, scales with the parameter $Q$ $=(1-b)\left(1-t^{2}\right)^{1 / 3}(t b)^{-2 / 3} G i^{-1 / 3}$, suggesting that a development of the Ginzburg-Landau free energy functional in terms of lowest Landau level (LLL) eigenfunctions is an appropriate starting point for a model description. ${ }^{19-24}$ Here, $t$ $\equiv T / T_{c}^{M F}$ with $T_{c}^{M F}$ the mean-field transition temperature, and $b \equiv B / B_{c 2}(T)$ with $B_{c 2}(T)=\Phi_{0} / 2 \pi \xi^{2}(T)$ the upper critical field. An assessment of the experimental result shows that in $\mathrm{YBa}_{2} \mathrm{Cu}_{3} \mathrm{O}_{7-\delta}$, fluctuations of the order parameter amplitude lower the pinning energy with respect to the expected meanfield value. In $\mathrm{K}_{x} \mathrm{Ba}_{1-x} \mathrm{BiO}_{3}$, with small $G i \sim 10^{-5}$, thermal fluctuations are unimportant and pinning subsists up to the superconducting-to-normal state transition.

\section{EXPERIMENTS AND RESULTS}

\section{A. Samples}

Experiments were done on a series of untwinned and lightly twinned $\mathrm{YBa}_{2} \mathrm{Cu}_{3} \mathrm{O}_{7-\delta}$ single crystals, grown by the flux method in Au crucibles, and subsequently annealed in oxygen in Pt tubes. ${ }^{25}$ Measurements on $\mathrm{K}_{x} \mathrm{Ba}_{1-x} \mathrm{BiO}_{3}$ were made on a crystal with $x=0.35$, grown by electrocrystallization. The crystals were irradiated during different runs at the Grand Accélérateur National d'Ions Lourds (GANIL) in Caen, France. The $\mathrm{YBa}_{2} \mathrm{Cu}_{3} \mathrm{O}_{7-\delta}$ crystals were irradiated with $5.8 \mathrm{GeV} \mathrm{Pb}$ ions; for all but one crystal, the beam was aligned parallel to the $c$ axis. The final crystal (hereafter referred to as "Y30") was irradiated at an angle of $30^{\circ}$ with respect to the $c$ axis, to a fluence of $1 \times 10^{11}$ ions $\mathrm{cm}^{-2}$, which corresponds to a matching field $B_{\phi} \equiv \Phi_{0} n_{d}=2 \mathrm{~T}$. The $\mathrm{K}_{0.35} \mathrm{Ba}_{0.65} \mathrm{BiO}_{3}$ crystal was irradiated with $7.2 \mathrm{GeV}$ Ta ions. The irradiation produced continuous amorphous columnar defects of radius $c_{0} \approx 3.5 \mathrm{~nm}$. The irreversibility line $T_{i r r}(H)$ of all crystals was measured as the onset temperature of the third harmonic of the ac transmittivity $T_{H 3},{ }^{26,27}$ with the dc field aligned parallel to the defect direction. The irreversibility line of the "Y30" and $\mathrm{K}_{0.35} \mathrm{Ba}_{0.65} \mathrm{BiO}_{3}$ crystal was also obtained from torque magnetometry (see Table II).

\section{B. Torque measurements}

Apart from the characterization of the pinning energy, torque measurements were also used to obtain the superconducting parameters of the two compounds under study (see Table I). The measurements on $\mathrm{YBa}_{2} \mathrm{Cu}_{3} \mathrm{O}_{7-\delta}$ were performed on the same twinned single crystal (Y30) as that used in Ref. 28. The microtorque setup ${ }^{28}$ was improved by the adjunction of a secondary magnetic field perpendicular to the main one, so that the plane in which the field is rotated could be chosen arbitrarily. The angle $\vartheta$ between this plane and the defect direction could be set with a resolution better than $0.1^{\circ}$. We set $\vartheta$ to zero $\pm 0.1^{\circ}$ by maximizing the pinning measured by the torque irreversibility along the defect direction (Fig. 1).

The mean-field upper critical field line $B_{c 2}(T)$ was located using the slope of the equilibrium torque per unit volume. For small angles $\theta$ between the applied field and the $c$ axis direction, this is given by

$$
d \Gamma / d \theta \simeq H M_{\perp}(H),
$$

where $M_{\perp}$ is the magnetization for the field applied along the $c$ axis. ${ }^{29-31}$ A plot of $H^{-1} d \Gamma / d \theta$, shown in Fig. 2, represents $M_{\perp}(H)$. For the lower temperatures, $H^{-1} d \Gamma / d \theta$ depends linearly on $T$, in agreement with the (mean-field) Abrikosov formula 
TABLE II. Characteristics of single crystals used in this study. All crystals were either untwinned or twinned with a single twin boundary (TB) orientation.

\begin{tabular}{|c|c|c|c|c|c|c|}
\hline & $\begin{array}{l}\mathrm{YBa}_{2} \mathrm{Cu}_{3} \mathrm{O}_{7-\delta} \\
\text { pristine }\end{array}$ & $B_{\phi}=1 \mathrm{~T}$ & $\begin{array}{c}\mathrm{YBa}_{2} \mathrm{Cu}_{3} \mathrm{O}_{7-\delta} \\
B_{\phi}=2 \mathrm{~T}\end{array}$ & $B_{\phi}=5 \mathrm{~T}$ & $\begin{array}{c}\mathrm{YBa}_{2} \mathrm{Cu}_{3} \mathrm{O}_{7-\delta} \\
B_{\phi}=2 \mathrm{~T} \\
30^{\circ}\end{array}$ & $\begin{array}{c}\mathrm{K}_{0.35} \mathrm{Ba}_{0.65} \mathrm{BiO}_{3} \\
B_{\phi}=2 \mathrm{~T}\end{array}$ \\
\hline Dimensions $\left(\mu \mathrm{m}^{3}\right)$ & $\begin{array}{c}330 \times 400 \\
\times 20\end{array}$ & $\begin{array}{c}\text { Triangle of base } 650, \\
\text { height } 530 \text {, thickness } 20\end{array}$ & $\begin{array}{l}430 \times 510 \\
\times 20\end{array}$ & $\begin{array}{l}200 \times 450 \\
\times 20\end{array}$ & $\begin{array}{c}130 \times 337 \times 18 \\
\text { "Y30" }\end{array}$ & $\begin{array}{c}120 \times 45 \\
\times 30\end{array}$ \\
\hline Description & $\begin{array}{l}\text { Untwinned } \\
\text { rectangle }\end{array}$ & 16 TB's \| base & $\begin{array}{l}\text { TB's spaced } \\
\text { by } 10 \mu \mathrm{m}\end{array}$ & twinned & $\begin{array}{l}\text { TB's spaced } \\
\text { by } 5 \mu \mathrm{m}\end{array}$ & $\begin{array}{c}\text { tracks } \| \\
\text { long dimension }\end{array}$ \\
\hline$T_{C_{n}}^{\max }(H=0)(\mathrm{K})$ & 93.1 & 92.4 & 92.1 & 91.0 & $*$ & $*$ \\
\hline$T_{i r r}^{p}(H=0)(\mathrm{K})$ & 93.1 & 92.5 & 92.0 & 90.8 & 91.3 & 31.5 \\
\hline$T_{c}^{M F}(\mathrm{~K})$ & 93.1 & 93.1 & 93.1 & 93.1 & 92.3 & 32.4 \\
\hline
\end{tabular}

$$
-M_{\perp} \approx \frac{\varepsilon_{0}}{\beta_{A} \Phi_{0}}(1-b) \quad(b \lesssim 1)
$$

with $\beta_{A}=1.16$. According to Eq. (2), the zero intercepts of $H^{-1} d \Gamma / d \theta$ correspond to $T_{c}(H)$, i.e., the $B_{c 2}(T)$ line. A plot of the zero intercepts for different fields yields the slope $d B_{c 2} / d T=-2 \mathrm{TK}^{-1}$ and $\xi_{a b}(0)=1.37 \mathrm{~nm}$, while dividing out the field dependence $1-b$ gives the line energy $\varepsilon_{0}(T)$. The inset (b) of Fig. 2 shows that $\varepsilon_{0}(T)$ does not depend on the field, as it should. The results were checked by plotting the scaled magnetization $H^{-1}(d \Gamma / d \theta)(t b)^{-2 / 3}$ as a function of the LLL parameter $Q$, see inset (c) of Fig. 2. The magnetization could be scaled using the experimental $d B_{c 2} / d T=-2 \mathrm{TK}^{-1}$ and the mean-field critical temperature $T_{c}^{M F}=92.3 \mathrm{~K}$. The linear portion of the $H^{-1} d \Gamma / d \theta$ curves falls in the regime $Q>10$, well outside the fluctuation-dominated region. The $B_{c 2}(T)$ line, plotted in Fig. 4, the $\xi_{a b}$ value deduced from it, and $d \varepsilon_{0} / d T$, which yields the penetration depth extrapolated to zero temperature $\lambda_{a b}(0)=120 \mathrm{~nm}$, agree well with the results in the literature. ${ }^{32}$

As was first shown in Ref. 28, to the best of our knowledge, and reproduced in the inset of Fig. 3, the equilibrium torque features a step of magnitude $\Gamma_{0}$ at the angle at which

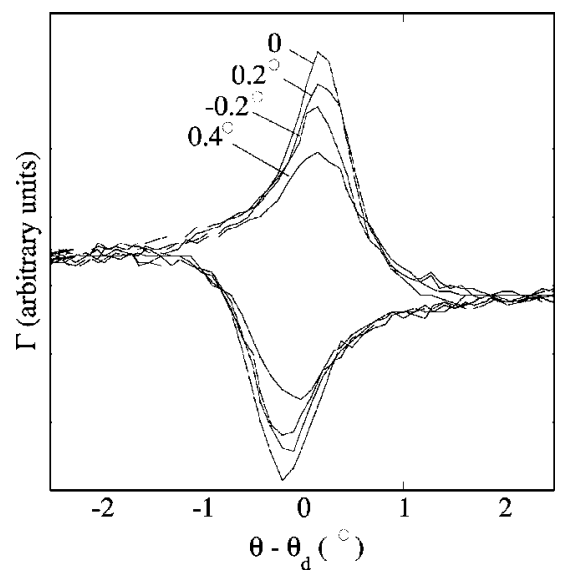

FIG. 1. $\mathrm{YBa}_{2} \mathrm{Cu}_{3} \mathrm{O}_{7-\delta}$ : Torque signal for rotation angles $\theta$ close to the irradiation direction $\theta_{d}$, for different angles $\vartheta$ between the plane of rotation of the magnetic field and the defects $(H=20 \mathrm{kOe}$, $T=88 \mathrm{~K})$ the field orientation coincides with the defect direction $\theta$ $=\theta_{d}$. On both sides of the step, the torque signal depends linearly on the field angle, allowing $\Gamma_{0}$ to be determined from the extrapolation of the torque to $\theta-\theta_{d}=0$ (Inset to Fig. 3 ). The main panel of Fig. 3 shows $\Gamma_{0}$ as function of temperature, for fields $0.5 B_{\phi}<\mu_{0} H<2 B_{\phi}$. The torque step decreases approximately linearly with temperature, with a roughly field-independent slope $d \Gamma_{0} / d T=-65 \pm 5 \mathrm{~J} \mathrm{~m}^{-3} \mathrm{~K}^{-1}$, and vanishes at the onset temperature $T_{k}(H)$ [or, conversely, at the field $\left.H_{k}(T)\right]$. The locus of $T_{k}(H)$, as well as the irreversibility line $T_{i r r}(H)$ and the $B_{c 2}(T)$ line, are displayed in Fig. 4. Additional measurements on samples with $B_{\phi}=1 \mathrm{~T}$ and $B_{\phi}=0.4 \mathrm{~T}$ indicate that $T_{k}(H)$ depends weakly on the irradiation dose in this range of $B_{\phi}$.

Concerning $\mathrm{K}_{0.35} \mathrm{Ba}_{0.65} \mathrm{BiO}_{3}$, a discontinuity in the reversible torque signal, superimposed to some irreversibility along the track direction, can be put into evidence in a way similar to $\mathrm{YBa}_{2} \mathrm{Cu}_{3} \mathrm{O}_{7-\delta}$. The torque step again depends roughly linearly on temperature, with slope $d \Gamma_{0} / d T \approx-6 \pm 0.5$ $\times 10^{2} \mathrm{~J} \mathrm{~m}^{-3} \mathrm{~K}^{-1}$ (Fig. 5). The $T_{k}(H)$ line is found to lie slightly above $T_{i r r}(H)$ (Fig. 6).

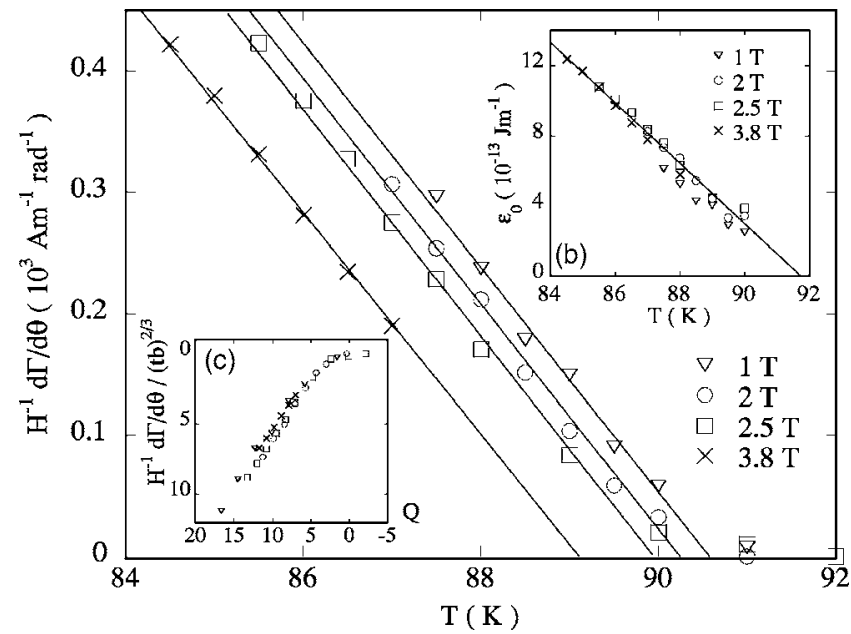

FIG. 2. $\mathrm{YBa}_{2} \mathrm{Cu}_{3} \mathrm{O}_{7-\delta}$ : Slope $\partial \Gamma / \partial \theta$ of the torque signal for field along the $c$ axis, divided by field. Lines are linear fits extrapolating to $T_{c}(H)$. Inset (b): line energy $\varepsilon_{0}(T)$ obtained by dividing out the field dependence from the data from the data in the main panel. Inset (c): Scaled magnetization $H^{-1} d \Gamma / d \theta /(t b)^{2 / 3}$ as function of the LLL parameter $Q=(1-b)\left(1-t^{2}\right)^{1 / 3} /(t b)^{2 / 3} G i^{1 / 3}$. 


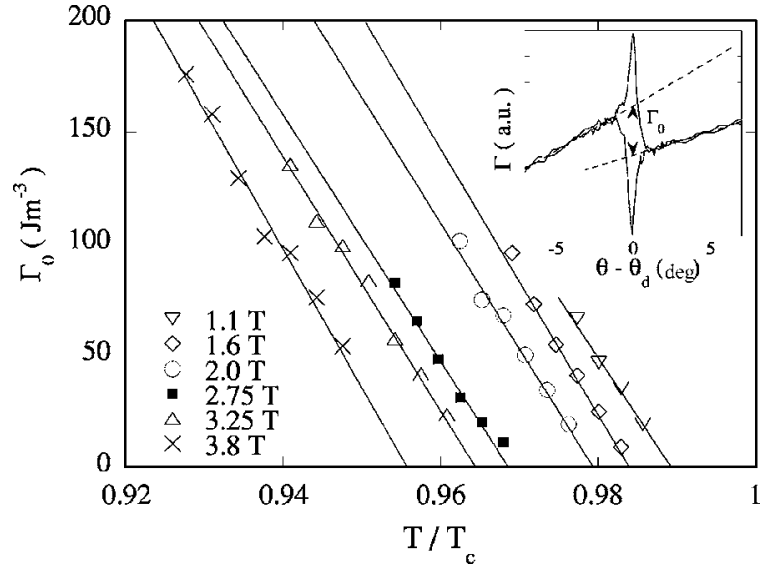

FIG. 3. $\mathrm{YBa}_{2} \mathrm{Cu}_{3} \mathrm{O}_{7-\delta}$ : magnitude of the torque discontinuity at field alignment with the defect direction $\theta_{d}$. Straight lines represent the extrapolation of the torque discontinuity to $T_{k}(H)$. The inset shows the torque curves for both directions of rotation at $\mu_{0} H$ $=2 \mathrm{~T}$ and $T=0.97 T_{c}$. It also shows the determination of the torque discontinuity $\Gamma_{0}$ by extrapolation of the linear signal to $\theta=0$.

For both compounds, the temperature and field dependence of $\Gamma_{0}$ is found to be parametrized, at all but the lowest measuring fields $\left(\mu_{0} H=1.1 \mathrm{~T}\right.$ for Y30, $0.75 \mathrm{~T}$ for $\mathrm{K}_{0.35} \mathrm{Ba}_{0.65} \mathrm{BiO}_{3}$ ), by the LLL parameter $Q$ [see Figs. 8(a) and $8(\mathrm{~b})]$. All torque step data trace the same curve when divided by the free energy density $k_{B} T B / \Phi_{0} \xi(0)$ and plotted vs $Q$, assuming the mean-field transition temperature to be $T_{c}^{M F}$ $=93.1 \mathrm{~K}$ in $\mathrm{YBa}_{2} \mathrm{Cu}_{3} \mathrm{O}_{7-\delta}$, and $32.4 \mathrm{~K}$ in $\mathrm{K}_{0.35} \mathrm{Ba}_{0.65} \mathrm{BiO}_{3}$. The condition for thermodynamic quantities to depend on the

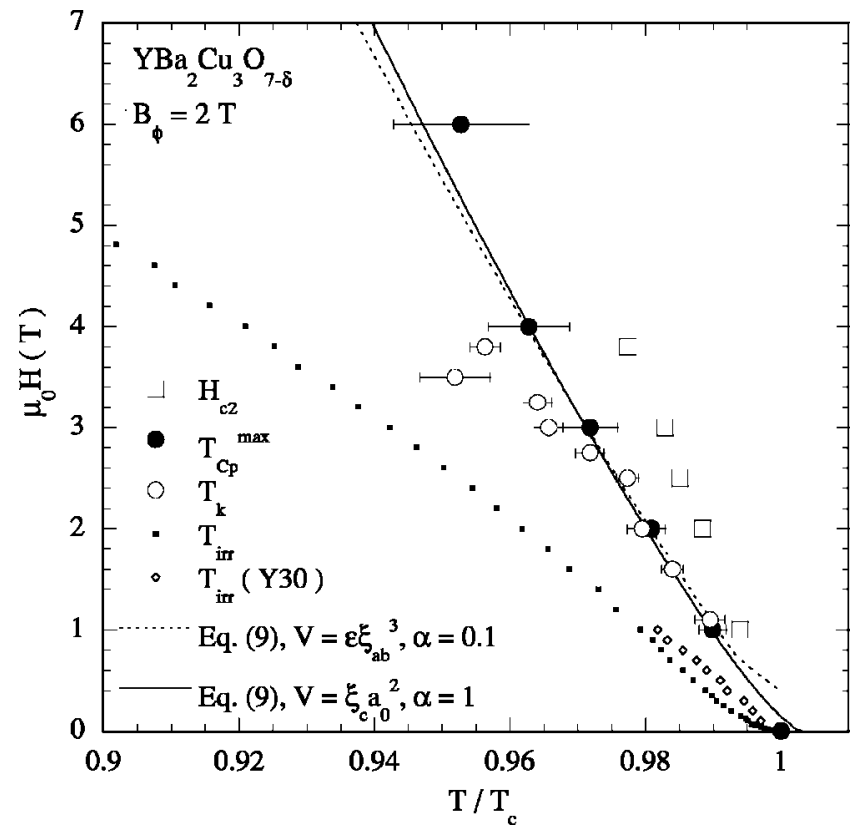

FIG. 4. $\mathrm{YBa}_{2} \mathrm{Cu}_{3} \mathrm{O}_{7-\delta}$ : Comparison of the $H_{c 2}(T)$ line $(\square)$, $T_{C p}^{\max }(H)(\bullet), T_{k}(H)(\circ)$, and $T_{i r r}(H)(\bullet \diamond)$ for the two crystals with $n_{d}=1 \times 10^{11}$ tracks $\mathrm{cm}^{-2}\left(B_{\phi}=2 \mathrm{~T}\right)$. The drawn line denotes the locus of $\left|F_{n}-F_{s}(B)+n_{t} U_{p}(B)\right|=\alpha k_{B} T / V$, with $V=\varepsilon a_{0}^{2} \xi_{a b}$ and $\alpha=1$ (see text). The dotted line shows the same, but choosing $V=\varepsilon \xi_{a b}^{3}$ and $\alpha=0.1$.

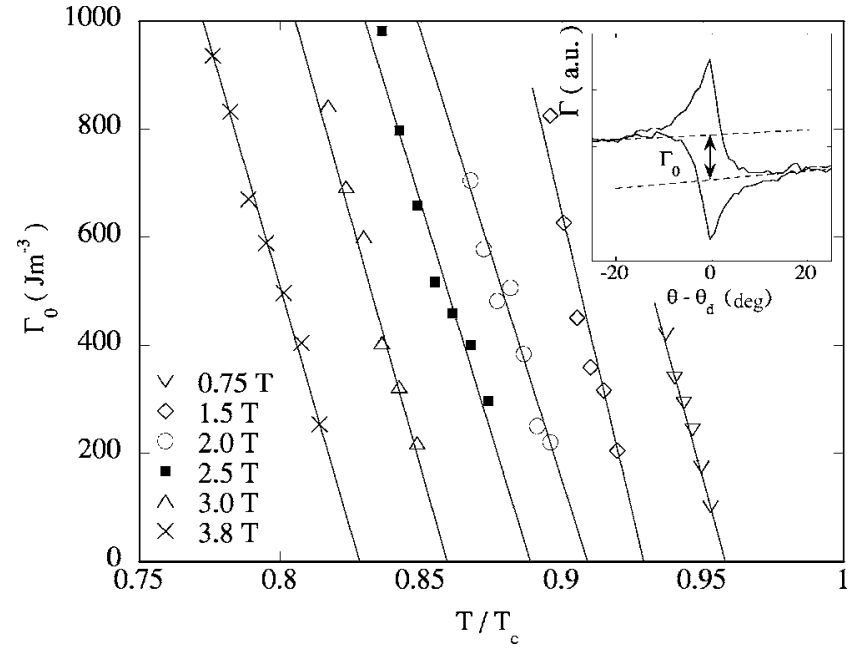

FIG. 5. $\mathrm{K}_{0.35} \mathrm{Ba}_{0.65} \mathrm{BiO}_{3}$ : Amplitude of the torque discontinuity $\Gamma_{0}$ for field alignment with the defect direction $\theta_{d}$. Straight lines show the extrapolation of the torque step to $T_{k}(H)$. The inset shows the torque curves for both directions of rotation and the determination of the torque jump as the difference between the linear extrapolations of the torque from high angle to $\theta=0$ (dotted lines). The applied field $\mu_{0} H=1.25 \mathrm{~T}$, the temperature $T=0.92 T_{c}$.

sole parameter $Q$ is that the Landau level splitting $\sim 2 T_{c}^{M F} h$ be greater than the strength of fluctuations $T_{c}^{M F}(2 G i)^{1 / 3}(h t)^{2 / 3}$ [with $\quad h \equiv 2 \pi \xi^{2}(0) B / \Phi_{0}=B / T_{c}^{M F} /\left(\partial B_{c 2} / \partial T\right)_{T=T_{c}^{M F}}$ $\left.=\left(\partial b^{-1} / \partial t\right)_{t=1}^{-1}\right] \cdot{ }^{33}$ In $\mathrm{YBa}_{2} \mathrm{Cu}_{3} \mathrm{O}_{7-\delta}$, at the temperatures under investigation, this condition is satisfied for fields larger than $1 \mathrm{~T}$. The failure of the low-field data to conform to the scaling might thus be due to Landau level degeneracy. In

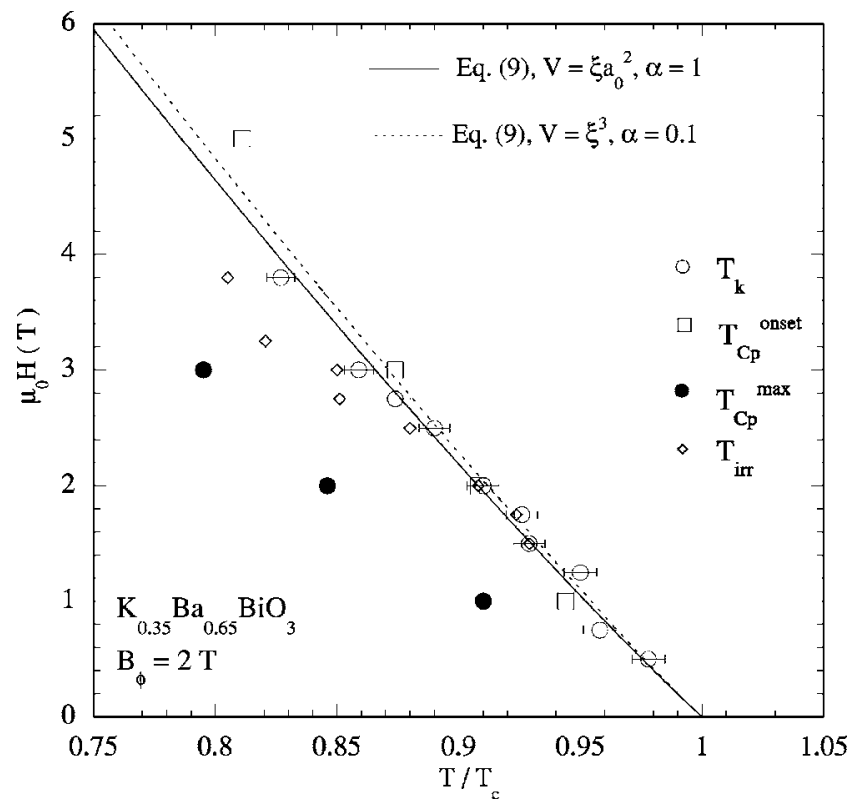

FIG. 6. $\mathrm{K}_{0.35} \mathrm{Ba}_{0.65} \mathrm{BiO}_{3}, B_{\phi}=2 \mathrm{~T}$ : Comparison of the specific anomaly heat onset temperature $T_{C p}^{\text {onset }}(H)(\square)$, the specific-heat maximum $T_{C p}^{\max }(H)(\bullet), T_{k}(H)(\bigcirc)$, and $T_{i r r}(H)(\diamond)$. The drawn line denotes the locus of $\left|F_{s}(B)+n_{t} U_{p}(B)-F_{n}\right|=\alpha k_{B} T / V$, with $V$ $=\varepsilon a_{0}^{2} \xi$ and $\alpha=1$ (see text). The dotted line shows the same, but choosing $V=\xi^{3}$ and $\alpha=0.1$. 
$\mathrm{K}_{0.35} \mathrm{Ba}_{0.65} \mathrm{BiO}_{3}$, however, the LLL condition is supposed to be satisfied at all fields investigated. The fact that the lowfield data do not conform to the LLL scaling in this compound either, suggest another origin of the breakdown of scaling. Namely, the intrinsic inhomogeneity introduced by the randomly positioned columnar defects leads to a spread in local field-dependent critical temperatures to which the superconductor is sensitive at fields $B \leqq B_{\phi} \cdot{ }^{10,12}$ As a result, the effective $T_{c}$ is not the real critical temperature but an average quantity (with a lower value) determined by the defect distribution. This effect was previously put into evidence in heavy-ion-irradiated $\mathrm{Bi}_{2} \mathrm{Sr}_{2} \mathrm{CaCu}_{2} \mathrm{O}_{8+\delta}$ : at fields $0.2 B_{\phi}$ $<B<B_{\phi}$ the magnetization was found to approximately follow the LLL scaling relation but with an effective critical temperature $2 \mathrm{~K}$ lower than the mean-field $T_{c}$ of the pristine sample. We suggest that a similar effect is responsible for the downward shift of the present $1 \mathrm{~T}$ data in $\mathrm{Y} 30$, and the $0.75 \mathrm{~T}$ data on the $\mathrm{K}_{0.35} \mathrm{Ba}_{0.65} \mathrm{BiO}_{3}$ crystal, as well as for the difference in $T_{c}^{M F}$ deduced for Y30 for fields along the tracks and fields along the $c$ axis (in the latter case, the field component along the defects is smaller).

\section{Specific heat}

Specific-heat measurements have been performed on all $\mathrm{YBa}_{2} \mathrm{Cu}_{3} \mathrm{O}_{7-\delta}$ crystals (see Table II). The same measurement technique was employed as in Ref. 13. Well-defined specificheat anomalies were observed in all crystals, in spite of the use of large irradiation doses (up to $B_{\phi}=5 \mathrm{~T}$ ).

In the pristine sample, the amplitude of the zero-field superconducting jump was of the order of $4 \%$ of the total specific heat, attesting to its very high quality. The anomaly presents the typical shape of the superconducting transition in the presence of strong thermal fluctuations for $H=0$, broadens for increasing $H$, and is shifted towards lower temperature (see Fig. 7). Sharp vortex lattice melting peaks are observed for $1 \mathrm{~T}<H<6 \mathrm{~T}$ (see the inset of Fig. 7). ${ }^{9,34}$

The presence of the amorphous columnar defects reduces the absolute temperature $T_{C_{p}}^{m a x}$ at which the specific-heat maximum occurs in zero field (see Table II). ${ }^{35}$ A lowering of the critical temperature after heavy-ion irradiation may occur as a result of "self-doping" of the intercolumn space by $\mathrm{O}$ ions expelled from the tracks, ${ }^{36,37}$ but no such effect was reported for $\mathrm{YBa}_{2} \mathrm{Cu}_{3} \mathrm{O}_{7-\delta}$. Rather, the columns may act by reducing the average zero field $T_{c}$ at which the long range superconducting order can set in. ${ }^{10,12}$

Turning to the specific-heat data in nonzero fields, we find that the columnar defects increase the (reduced) temperature $T_{C_{p}}^{\max }(H) / T_{c}$ of the $C_{p}$ maximum (Fig. 7). Furthermore, the specific-heat curves in magnetic fields become sharper after irradiation. Apparently, columnar defects suppress order parameter fluctuations in a magnetic field, even though the effect in $\mathrm{YBa}_{2} \mathrm{Cu}_{3} \mathrm{O}_{7-\delta}$ is weaker than that previously found in $\mathrm{K}_{x} \mathrm{Ba}_{1-x} \mathrm{BiO}_{3} \cdot{ }^{13,14}$ No vortex lattice melting anomaly is observed in the irradiated crystals, nor is any other anomaly at, e.g., the irreversibility line.

Figure 4 shows that in $\mathrm{YBa}_{2} \mathrm{Cu}_{3} \mathrm{O}_{7-\delta}$, the maximum of the specific heat systematically coincides with the temperature $T_{k}$ at which the torque signal from vortex pinning by the

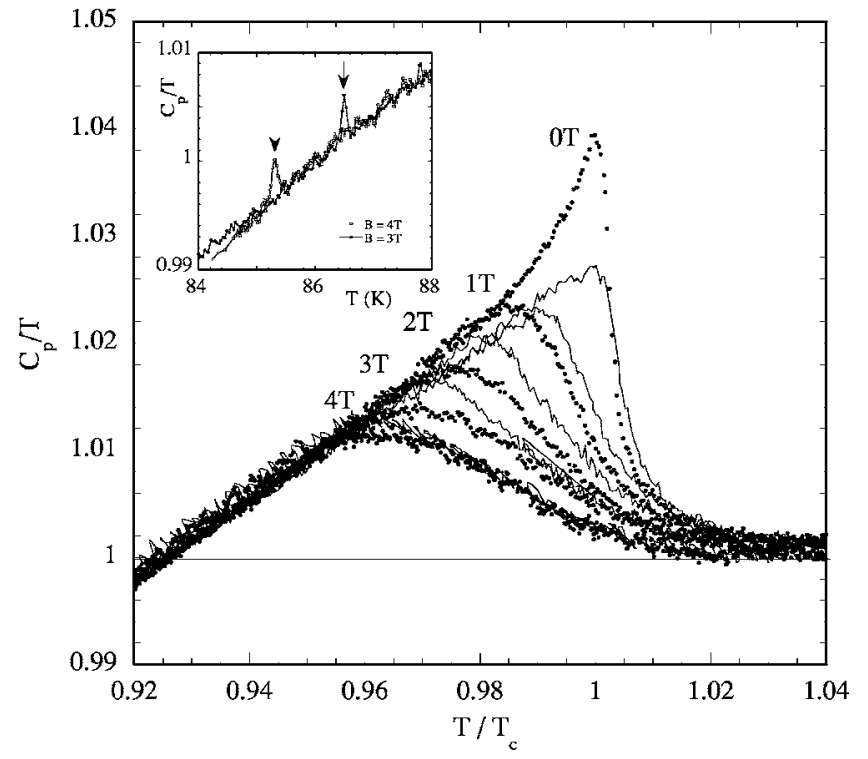

FIG. 7. $\mathrm{YBa}_{2} \mathrm{Cu}_{3} \mathrm{O}_{7-\delta}$ : Normalized specific heat data on a pristine crystal (points), and on a crystal with $n_{d}=1 \times 10^{11} \mathrm{~cm}^{-2}$, i.e., $B_{\phi}=2 \mathrm{~T}$ (thin lines). In order to compare the two data sets, the temperature has been rescaled to $t=T / T_{c}$, and the specific heat to the normal state (phonon) contribution at $T_{c}$. The temperature dependence due to phonons was subtracted. The inset shows an enlargement of the specific heat curve measured on the pristine crystal in fields of 3 and $4 \mathrm{~T}$.

columnar defects disappears. Thus, vortex pinning by the columns is responsible for the upward shift of the superconducting transition. This is obvious when one considers the shift for different columnar defect densities $n_{d}$ (matching fields $B_{\phi}$ ). Figure 9 shows that a higher density of columns leads to a higher $T_{C_{p}}^{\max }$.

Figure 6 traces the onset temperature $T_{C_{p}}^{\text {onset }}(H)$ of the specific-heat anomaly measured in $\mathrm{K}_{0.35} \mathrm{Ba}_{0.65} \mathrm{BiO}_{3}, 13,14$ along with the $T_{k}(H)$ - and $T_{i r r}(H)$ lines obtained here. In the bismuthate compound the two latter lines coincide with the specific-heat onset, which is much sharper than the one in $\mathrm{YBa}_{2} \mathrm{Cu}_{3} \mathrm{O}_{7-\delta}$. This attests to the fact that in this compound, the effect of the columnar defects is important enough to not only remain present up to $H_{c 2}(T)$, but to actually determine the position of the upper critical field line (see also Refs. 13 and 14).

In the LLL scenario, the specific heat in magnetic fields should behave as $C_{p} / T=F(Q)$, with $F$ a universal scaling function. ${ }^{20,22}$ Figures $8(\mathrm{c})$ and $8(\mathrm{~d})$ shows that the location in the $(H, T)$ plane of characteristic features of $C_{p}$ are satisfactorily described by $Q=$ const. For example, vortex lattice melting in the pristine crystal occurs at constant $Q=7.9,{ }^{23}$ and the position of the maximum at $Q=3.2$. However, as in Refs. 20, 38, and 39, the magnitude of $\Delta C_{p} / T=C_{p}-C_{N}$ does not scale as expected, whatever the choice for the normal contribution $\left(C_{N}\right)$ [see Figs. 8(c) and 8(d)].

\section{DATA ANALYSIS AND DISCUSSION}

\section{A. Determination of the pinning energy}

The torque exerted by a perpendicular magnetic-field component on the trapped vortices allows one to measure the 

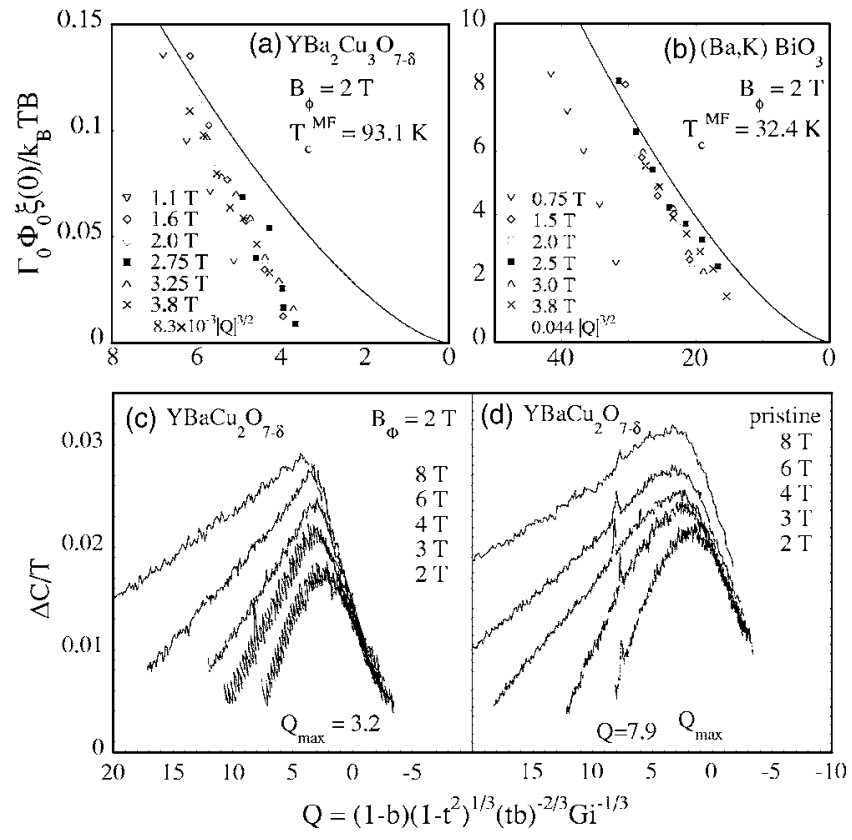

FIG. 8. Lowest Landau level scaling of the torque (a) and (b) and specific heat data (c) and (d). (a) Scaled torque jump, measured on $\mathrm{YBa}_{2} \mathrm{Cu}_{3} \mathrm{O}_{7-\delta}$ crystal $\mathrm{Y} 30$, versus LLL parameter $Q$ (see text). The parameter $T_{c}^{M F}=93.1 \mathrm{~K}$. (b) Scaled torque jump, measured on the $\mathrm{Ba}_{0.65} \mathrm{~K}_{0.35} \mathrm{BiO}_{3}$ crystal with $B_{\phi}=2 \mathrm{~T}$, versus $Q$, with $T_{c}^{M F}$ $=32.4 \mathrm{~K}$. (c) $C / T$ versus $Q$ for irradiated $\mathrm{YBa}_{2} \mathrm{Cu}_{3} \mathrm{O}_{7-\delta}$ with $B_{\phi}$ $=2 \mathrm{~T}$. (d) $C / T$ versus $Q$ for pristine $\mathrm{YBa}_{2} \mathrm{Cu}_{3} \mathrm{O}_{7-\delta}$.

energy gain due to vortex localization on the columnar defects, ${ }^{41}$ i.e., the pinning energy. Writing out the torque step $\Gamma_{0} \simeq H_{\perp} M_{\|}-\left.H_{\|} M_{\perp}\right|_{\theta=\theta_{d}}=-H_{\|} \partial G / \partial B_{\perp}$ shows that it is a measurement of the energy per unit length $E_{k}=\Phi_{0} \partial G / \partial B_{\perp}$ of vortex kinks joining different defects $(G$ is the Gibbs free energy). In the limit of isolated flux lines, $\Gamma_{0} \approx 2 E_{k} / a_{0}^{2}$, where $a_{0} \simeq\left(\Phi_{0} / B\right)^{1 / 2}$ is the flux line spacing. The kink energy $E_{k}=\left(\frac{1}{2} U_{p} \varepsilon_{l}\right)^{1 / 2}$, where $U_{p}$ is the pinning energy of the defect per unit length, and $\varepsilon_{l}$ is the vortex line tension. ${ }^{3}$ For a single-vortex line, the pinning energy can be written as $^{3}$

$$
\begin{gathered}
U_{p}=\frac{k_{B}^{2} T^{2}}{4 \varepsilon_{l} \xi^{2}} \beta \quad(\beta \gg 1) \\
U_{p}=\frac{k_{B}^{2} T^{2}}{4 \varepsilon_{l} \xi^{2}} \beta \exp (-\pi / \sqrt{\beta}) \quad(\beta \ll 1) .
\end{gathered}
$$

The pinning strength $\beta$ can be approximated as $\beta_{\text {core }}$ $=\left(c_{0}^{2} \varepsilon_{0} \varepsilon_{l} / k_{B}^{2} T^{2}\right)(1-b)^{2}$ for vortex core pinning (for $c_{0} \ll \xi$ ) and $\beta=\beta_{e m}=\left(\xi^{2} \varepsilon_{0} \varepsilon_{l} / k_{B}^{2} T^{2}\right)(1-b)$ for electromagnetic pinning $\left(c_{0} \gg \xi\right){ }^{3}$ The exponential factor in Eq. (4) expresses the reduction of the pinning energy due to vortex line wandering when $\beta \ll 1$. As a consequence, the torque jump

$$
\Gamma_{0}=\frac{k_{B} T}{\sqrt{2} a_{0}^{2} \xi} \beta^{1 / 2} \quad(\beta \gg 1)
$$

$$
\Gamma_{0}=\frac{k_{B} T}{\sqrt{2} a_{0}^{2} \xi} \beta^{1 / 2} \exp \left(-\pi / \beta^{1 / 2}\right) \quad(\beta \ll 1)
$$

is a direct measure of the pinning strength $\beta$. Note that in the single-vortex limit, the dimensionless torque plotted in Figs. $8(\mathrm{a})$ and 8 (b) would directly correspond to the square-root of the pinning strength $\beta$.

In practice though, experiments are rarely carried out in the isolated vortex limit. At high fields, only a fraction $N_{t}$ $=1-\exp \left(-a_{0}^{2} n_{d}\right) \approx B_{\phi} / B$ of the vortices are trapped by the columns. Then we can write

$$
\Gamma_{0}=\frac{k_{B} T B_{\phi}}{\sqrt{2} \Phi_{0} \xi} \beta^{1 / 2} \quad(\beta \gg 1) ;
$$

taking $\beta=\beta_{\text {core }}$, we can rewrite this as the "mean-field expression"

$$
\frac{\Phi_{0} \xi(0)}{k_{B} T B} \Gamma_{0}=\frac{c_{0}}{4 \pi \xi(0)} \frac{B_{\phi}}{B_{c 2}(0)}|Q|^{3 / 2} \quad(\beta \gg 1) .
$$

In other words, in high fields the torque jump normalized by the energy density scale $k_{B} T / a_{0}^{2} \xi(0)$ is expected to follow an expression that only depends on the LLL scaling parameter $Q$. Note that the functional dependence on temperature and field following the LLL parameter is only followed provided vortex line wandering is not important $(\beta \gg 1)$. The exponential factor in Eq. (6) related to vortex line wandering would spoil the scaling, because its argument cannot be expressed as a function of $Q$. The irrelevance of line wandering is slightly surprising, for our experiments are carried out at high temperatures at which it is expected to be relevant. ${ }^{2,4,6}$ However, experiment unambiguously shows that this is not the case. In other words, a description of pinning in terms of vortex line fluctuations only (i.e., the London model) is not a good starting point for the description of vortex physics in heavy-ion-irradiated high temperature superconductors in Tesla fields, regardless of their anisotropy. The reason is that vortex line fluctuations can be interpreted as the result of superposing thermally generated vortex loops on regular, field-generated vortices. ${ }^{42}$ At high fields at which the LLL condition $4 B \xi^{2}(0) / \Phi_{0}>G i$ is satisfied, thermal vortices cannot be excited, as these imply Landau-level degeneracy. ${ }^{33}$ Rather, our data show that the fluctuation of the order parameter amplitude must be considered when describing pinning in the vortex liquid, as was already suggested in Ref. 40.

The importance of order parameter amplitude fluctuations to pinning becomes apparent when we compare expression (8) to the experimental data, see Figs. 8(a) and 8(b). For $\mathrm{YBa}_{2} \mathrm{Cu}_{3} \mathrm{O}_{7-\delta}$, the experimental torque lies below a putative $|Q|^{3 / 2}$-behavior (8). Note that the absolute value of the experimental torque is larger than expected: the prefactor in Eq. (8) $\left[c_{0} / 4 \pi \xi(0)\right] B_{\phi} / B_{c 2}(0)=5 \times 10^{-3}$. This discrepancy can be attributed to experimental uncertainty in the value of the effective track radius $c_{0}$ and $B_{c 2}(0)$. The temperature dependence of the experimental data is consistent with either an exponential $\left[a_{0}^{2} \xi(0) / k_{B} T\right] \Gamma_{0} \propto e^{-Q}$, or with a power-law drop $\left[a_{0}^{2} \xi(0) / k_{B} T\right] \Gamma_{0} \propto Q^{4}$, or with a polynomial in $Q$, but there is no sign of divergent behavior on approaching the irreversibility line. ${ }^{43}$ In $\mathrm{K}_{0.35} \mathrm{Ba}_{0.65} \mathrm{BiO}_{3}$, the experimental pinning 
energy is even larger. It closely follows $|Q|^{3 / 2}$ (8), indicating that fluctuations have little importance due to the small Ginzburg number of $\mathrm{K}_{0.35} \mathrm{Ba}_{0.65} \mathrm{BiO}_{3}$.

\section{B. Shift of the specific-heat maximum}

We finish by showing that vortex core pinning by the columnar defects, such as described by Eq. (8), leads to a correct estimate of the specific-heat shift in both $\mathrm{YBa}_{2} \mathrm{Cu}_{3} \mathrm{O}_{7-\delta}$ and $\mathrm{K}_{0.35} \mathrm{Ba}_{0.65} \mathrm{BiO}_{3}$, and may thus account for the free energy gain obtained from pinning. We adopt the procedure of Ref. 14, in which the field-angle dependence and the defect density-dependent shift of the onset temperature $T_{C_{p}}^{\text {onset }}(H)$ in $\mathrm{K}_{x} \mathrm{Ba}_{1-x} \mathrm{BiO}_{3}$ was well described quantitatively. To estimate $T_{C_{p}}^{\text {onset }}(H)$, we add the free energy change $n_{t} U_{p}(B)$ from pinning to the free energy difference $F_{n}$ $-F_{s}(B)=(1 / 2) \mu_{0} H_{c 2}^{2}(1-b)^{2}$ of the normal and superconducting states of the pristine material, and equate ${ }^{14,44}$

$$
\left|F_{n}-F_{s}(B)+n_{t} U_{p}(B)\right|=\alpha \frac{k_{B} T}{V} .
$$

Here $n_{t}=N_{t} a_{0}^{-2}$ is the areal density of vortices trapped on a columnar defect, $U_{p}$ is the average pinning energy per vortex per unit length, given by Eq. (3), $V=\varepsilon \xi^{3}$ is the coherence volume, and $\alpha \leq 1$. In Ref. 14, Eq. (9) was found to quantitatively reproduce all lines $\left[T_{i r r}(H), T_{k}(H), T_{C_{p}}^{\text {onset }}(H)\right]$ if one chooses $U_{p}$ to be given by the expression for electromagnetic pinning, Eq. (4) with $\beta=\beta_{e m}$ and $\alpha \approx 1$. There is some latitude in the choice of parameters: the dotted line in Fig. 6 shows that similar good agreement can be obtained with $\beta$ $=\beta_{\text {core }}$ and $\alpha=0.1$.

We find that the position of the $T_{C_{p}}^{\max }$ line in $\mathrm{YBa}_{2} \mathrm{Cu}_{3} \mathrm{O}_{7-\delta}$ can also be described in this way. The dotted line in Fig. 4 shows the solution of Eq. (9) with parameters for $\mathrm{YBa}_{2} \mathrm{Cu}_{3} \mathrm{O}_{7-\delta}$, the pinning energy as given by Eq. (4) with $\beta=\beta_{\text {core }}$ and $\alpha=0.1$.

Another approach is to choose the correlation volume $V$ $=\varepsilon a_{0}^{2} \xi$ rather than $\varepsilon \xi^{3}$. In the absence of columnar defects $\left(n_{t}=0\right)$, Eq. (9) can then be reduced to

$$
Q^{3 / 2}=4 \pi \sqrt{2} \alpha,
$$

which, for $\alpha=1.25(Q=7.9)$, perfectly describes the position of the vortex lattice melting line (see Fig. 9). In the presence of columnar defects, one again adds the free energy gain $n_{t} U_{p}$. Taking the same expressions $U_{p}=\left(c_{0} / 2 \xi\right)^{2} \varepsilon_{0}(1-b)^{2}$ and $n_{t}=B_{\phi} / \Phi_{0}$ used to derive Eq. (8), Eq. (9) becomes

$$
Q^{3 / 2}=\frac{4 \pi \sqrt{2} \alpha}{1+B_{\phi} / B_{c 2}(0)} .
$$

In other words, the criterion (9) takes the form " $Q=$ const," where the constant depends on the density of columnar defects. In order to describe the shift of the specific-heat maximum with $B_{\phi}$, we adopt the critical temperature $T_{c}^{M F}$ $=93.1 \mathrm{~K}$ obtained from the scaling of the torque and the specific heat, and evaluate

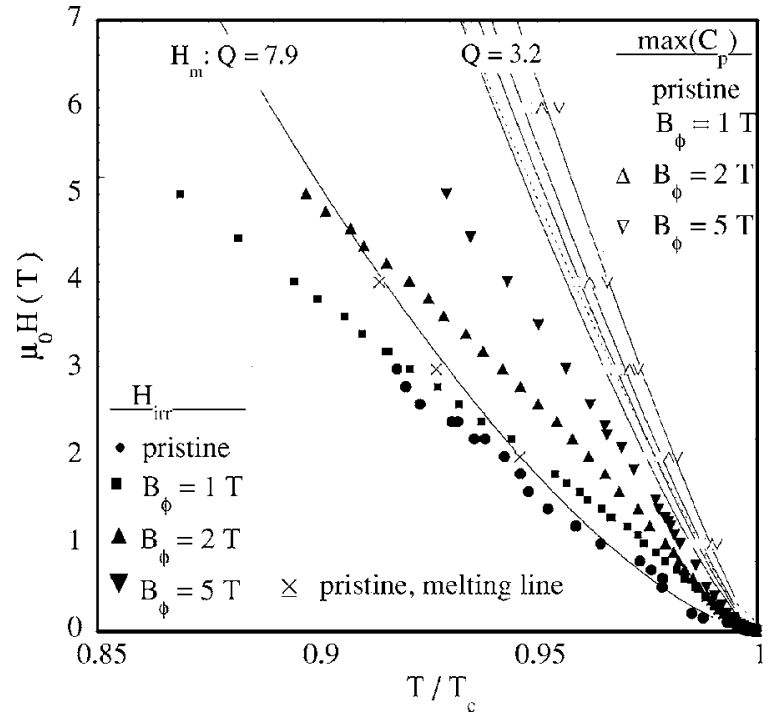

FIG. 9. $\mathrm{YBa}_{2} \mathrm{Cu}_{3} \mathrm{O}_{7-\delta}$ : Closed symbols denote the irreversibility lines $H_{i r r}(T)$ determined from the onset of the third harmonic transmittivity, for single crystals with $B_{\phi}=0(\bullet), B_{\phi}=1 \mathrm{~T}(\mathbf{\square}), B_{\phi}=2 \mathrm{~T}$ $(\triangle)$, and $B_{\phi}=5 \mathrm{~T}(\nabla)$. Open symbols $(\bigcirc, \square, \triangle, \nabla)$ correspond to the $T_{C_{p}}^{\max }$ line tracing the temperature of the specific-heat maximum, for the same crystals. The drawn lines denote the solution of Eq. (12), for matching fields $B_{\phi}=0,1,2$, and $5 \mathrm{~T}$ (see text). The dotted line shows an evaluation of criterion (9), choosing $V=\varepsilon \xi_{a b}^{3}$ and $\alpha$ $=0.1$, for $B_{\phi}=1 \mathrm{~T}$ only. The crossed squares depict the vortex lattice melting line of the pristine crystal, which is well described by $Q=7.9$.

$$
Q=3.2 /\left[1+B_{\phi} / B_{c 2}(0)\right]^{2 / 3}
$$

so as to recover the correct position of the specific-heat maximum for $B_{\phi}=0$ (see Fig. 8). The dependence (12) well describes the upwards shift of the specific-heat maximum in a magnetic field (Fig. 9). Thus, we find that the incorporation of the pinning energy in the free energy difference between the normal and superconducting states satisfactorily describes the evolution of the specific heat as a function of defect density.

\section{SUMMARY AND CONCLUSIONS}

Thermodynamic measurements in the vortex liquid of heavy-ion-irradiated $\mathrm{YBa}_{2} \mathrm{Cu}_{3} \mathrm{O}_{7-\delta}$ show that, as in $\mathrm{K}_{x} \mathrm{Ba}_{1-x} \mathrm{BiO}_{3}$, the reduction of the average free energy of the superconductor due to vortex pinning on columnar defects remains important all the way into the regime of strong order parameter amplitude fluctuations. Notably, the effect of pinning on the free energy is sufficient to shift the superconducting transition, as measured by the heat capacity, further upwards as the defect density increases. The scaling of the pinning energy with the LLL parameter $Q$ shows that pinning is affected by fluctuations of the overall order parameter amplitude. These should therefore be taken into account in any description of pinning in the vortex liquid-a model based on vortex line positional fluctuations only is bound to be inadequate. However, due to the lower Ginzburg number, order parameter fluctuations are much less important in the 
bismuthate superconductor than in $\mathrm{YBa}_{2} \mathrm{Cu}_{3} \mathrm{O}_{7-\delta}$.

This brings us to the final point, which is the Bose-glass transition. Our results indicate that depinning of vortices from columnar defects due to line wandering is not likely to be a very effective mechanism for this transition. On the other hand, fluctuations of the overall order parameter amplitude are also expected to become unimportant at, or near to, the Bose-glass transition. We note that, contrary to the melting line in the pristine crystals, the irreversibility line (and especially its high-field part) cannot be described as a line of constant $Q$ and therefore does not satisfy LLL scaling. We therefore suggest that another delocalization mechanism drives the Bose-glass transition. This could be, for example, the vortex delocalization due to variations of the pinning potential. Analogously to the electronic mobility in disordered semiconductors, the most weakly bound vortices could be delocalized at a much lower temperature than the bulk of the vortex matter. ${ }^{5}$ Another possibility is that the plastic properties of the vortex ensemble play a much more impor- tant role than hitherto considered. For example, the trapped vortex ensemble could constitute a polycrystal, vortex delocalization taking place on the grain boundaries. In both cases, another lower energy scale than the average pinning energy is involved in vortex delocalization at $T_{i r r}$, a scale that manifests itself through the large separation between $T_{i r r}$ and $\left[T_{k}(H), T_{C_{p}}(H)\right]$ lines in $\mathrm{YBa}_{2} \mathrm{Cu}_{3} \mathrm{O}_{7-\delta}$.

The situation in cubic $\mathrm{K}_{x} \mathrm{Ba}_{1-x} \mathrm{BiO}_{3}$ seems to be different. We have shown that the disappearance of pinning by columnar defects in this compound happens indistinguishably close to the superconducting-to-normal boundary. It means that in this compound, vortices are neither depinned, nor delocalized at all, and the Bose-glass phase subsists up to the temperature at which superconductivity disappears altogether.

\section{ACKNOWLEDGEMENT}

We are very grateful to $\mathrm{F}$. Holtzberg for providing the $\mathrm{YBa}_{2} \mathrm{Cu}_{3} \mathrm{O}_{7-\delta}$ crystals.
${ }^{1}$ D. R. Nelson and V. M. Vinokur, Phys. Rev. Lett. 68, 2398 (1992); D. R. Nelson and V. M. Vinokur, Phys. Rev. B 48, 13060 (1993).

${ }^{2}$ A. V. Samoilov, M. V. Feigel'man, M. Konczykowski, and F. Holtzberg, Phys. Rev. Lett. 76, 2798 (1996); M. Konczykowski and A. V. Samoilov, ibid. 78, 1830 (1997).

${ }^{3}$ G. Blatter, M. V. Feigel'man, V. B. Geshkenbein, A. I. Larkin, and V. M. Vinokur, Rev. Mod. Phys. 66, 1125 (1994).

${ }^{4}$ L. Krusin-Elbaum, L. Civale, G. Blatter, A. D. Marwick, F. Holtzberg, and C. Feild, Phys. Rev. Lett. 72, 1914 (1994); A. V. Samoilov and M. Konczykowski, ibid. 75, 186 (1995); L. Krusin-Elbaum, G. Blatter, and L. Civale, ibid. 75, 187 (1995).

${ }^{5}$ A. I. Larkin and V. M. Vinokur, Phys. Rev. Lett. 75, 4666 (1995).

${ }^{6}$ G. Blatter and V. B. Geshkenbein, Vortex Matter, The Physics of Superconductors, Vol. 1: Conventional and High- $T_{c}$ Superconductors (Springer, Berlin, 2003), p. 726.

${ }^{7}$ H. Safar, P. L. Gammel, D. A. Huse, D. J. Bishop, J. P. Rice, and D. M. Ginsberg, Phys. Rev. Lett. 69, 824 (1992).

${ }^{8}$ W. K. Kwok, S. Fleshler, U. Welp, V. M. Vinokur, J. Downey, G. W. Crabtree, and M. M. Miller, Phys. Rev. Lett. 69, 3370 (1992)

${ }^{9}$ A. Schilling, R. A. Fisher, N. E. Phillips, U. Welp, D. Dasgupta, W. K. Kwok, and G. W. Crabtree, Nature (London) 382, 791 (1996).

${ }^{10}$ C. J. van der Beek, M. Konczykowski, T. W. Li, P. H. Kes, and W. Benoit, Phys. Rev. B 54, R792 (1996).

${ }^{11}$ C. J. van der Beek, M. Konczykowski, R. J. Drost, P. H. Kes, N. Chikumoto, and S. Bouffard, Phys. Rev. B 61, 4259 (2000).

${ }^{12}$ G. M. Braverman, S. A. Gredeskul, and Y. Avishai, Phys. Rev. B 65, 054512 (2002).

${ }^{13}$ C. Marcenat, S. Blanchard, J. Marcus, L. M. Paulius, C. J. van der Beek, M. Konczykowski, and T. Klein, Phys. Rev. Lett. 90, 037004 (2003).

${ }^{14}$ T. Klein, C. Marcenat, S. Blanchard, J. Marcus, C. Bourbonnais, R. Brusetti, C. J. van der Beek, and M. Konczykowski, Phys. Rev. Lett. 92, 037005 (2004).
${ }^{15}$ W. Jiang, N.-C. Yeh, D. S. Reed, U. Kriplani, D. A. Beam, M. Konczykowski, T. A. Tombrello, and F. Holtzberg, Phys. Rev. Lett. 72, 550 (1994).

${ }^{16}$ L. M. Paulius, J. A. Fendrich, W. K. Kwok, A. E. Koshelev, V. M. Vinokur, G. W. Crabtree, and B. G. Glagola, Phys. Rev. B 56, 913 (1997).

${ }^{17}$ J.-T. Kim, Y. K. Park, J.-C. Park, H. R. Lim, S. Y. Shim, D. H. Kim, W. N. Kang, J. H. Park, T. S. Hahn, S. S. Choi, W. C. Lee, J. D. Hettinger, and K. E. Gray, Phys. Rev. B 57, 7499 (1998).

${ }^{18}$ W. K. Kwok, L. M. Paulius, V. M. Vinokur, A. M. Petrean, R. M. Ronningen, and G. W. Crabtree, Phys. Rev. B 58, 14594 (1998).

${ }^{19}$ R. Ikeda, T. Ohmi, and T. Tsuneto, J. Phys. Soc. Jpn. 58, 1377 (1989).

${ }^{20}$ U. Welp, S. Fleshler, W. K. Kwok, R. A. Klemm, V. M. Vinokur, J. Downey, B. Veal, and G. W. Crabtree, Phys. Rev. Lett. 67, 3180 (1991).

${ }^{21}$ R. Šašik and D. Stroud, Phys. Rev. Lett. 75, 2582 (1995).

${ }^{22}$ D. Li and B. Rosenstein, Phys. Rev. Lett. 86, 3618 (2001); B. Rosenstein, Phys. Rev. B 60, 4268 (1999).

${ }^{23}$ D. Li and B. Rosenstein, Phys. Rev. B 65, 220504(R) (2002).

${ }^{24}$ G. P. Mikitik and E. H. Brandt, Phys. Rev. B 68, 054509 (2003).

${ }^{25}$ F. Holtzberg and C. Feild, Eur. J. Solid State Inorg. Chem. 27, 107 (1990).

${ }^{26}$ M. Konczykowski, Physica C 209, 247 (1993).

${ }^{27}$ C. J. van der Beek, M. Konczykowski, V. M. Vinokur, G. W. Crabtree, T. W. Li, and P. H. Kes, Phys. Rev. B 51, 15492 (1995).

${ }^{28}$ B. Hayani, S. T. Johnson, L. Fruchter, and C. J. van der Beek, Phys. Rev. B 61, 717 (2000).

${ }^{29}$ G. Le Bras, L. Fruchter, V. Vulcanescu, V. Viallet, A. Bertinotti, A. Forget, J. Hammann, F.-F. Marucco, and D. Colson, Physica C 271, 205 (1996).

${ }^{30}$ Zhidong Hao and J. R. Clem, Phys. Rev. B 43, 7622 (1991).

${ }^{31}$ A. Buzdin and D. Feinberg, Physica C 220, 74 (1994).

${ }^{32}$ J. E. Sonier, J. H. Brewer, and R. F. Kiefl, Rev. Mod. Phys. 72, 769 (2000). 
${ }^{33}$ R. Ikeda, J. Phys. Soc. Jpn. 64, 1683 (1995).

${ }^{34}$ F. Bouquet, C. Marcenat, E. Steep, R. Calemczuk, W. K. Kwok, U. Welp, G. W. Crabtree, R. A. Fisher, N. E. Phillips, and A. Schilling, Nature (London) 411, 448 (2001).

${ }^{35}$ C. J. van der Beek, T. Klein, C. Marcenat, R. Brusetti, A. Vestergren, M. Wallin, S. Teitel, and H. Weber (unpublished).

${ }^{36}$ A. Pomar, Z. Konstantinovic, L. Martel, Z. Z. Li, and H. Raffy, Phys. Rev. Lett. 85, 2809 (2000).

${ }^{37}$ M. Li, C. J. van der Beek, M. Konczykowski, H. W. Zandbergen, and P. H. Kes, Phys. Rev. B 66, 014535 (2002).

${ }^{38}$ T. Klein, A. Conde-Gallardo, I. Joumard, J. Marcus, C. J. van der Beek, and M. Konczykowski, Phys. Rev. B 61, R3830 (2000).
${ }^{39}$ E. Janod, A. Junod, K.-Q. Wang, G. Triscone, R. Calemczuk, and J.-Y. Henry, Physica C 234, 269 (1994); A. Junod, E. Bonjour, R. Calemczuk, J.-Y. Henry, J. Muller, G. Triscone, and J.-C. Vallier, ibid. 194-196, 1499 (1994).

${ }^{40}$ R. Ikeda, J. Phys. Soc. Jpn. 65, 3998 (1996).

${ }^{41}$ R. J. Drost, C. J. van der Beek, J. A. Heijn, M. Konczykowski, and P. H. Kes, Phys. Rev. B 58, R615 (1998).

${ }^{42}$ A. K. Nguyen and A. Sudbø, Phys. Rev. B 60, 15307 (1999).

${ }^{43}$ Z. Tešanovic and I. F. Herbut, Phys. Rev. B 50, 10389 (1994).

${ }^{44}$ J. R. Cooper, J. W. Loram, and J. M. Wade, Phys. Rev. B 51, 6179 (1995). 